\title{
Antenna Array Control via Integrated Optically- Activated Organic Semiconductor for S-Band Applications
}

\author{
Andre Sarker Andy ${ }^{1 *}$, James William Ewart Kneller $^{2}$, Rostyslav Dubrovka $^{1}$, Theo Kreouzis ${ }^{2}$, Robert Donnan ${ }^{1}$ \\ ${ }^{1}$ School of Electronic Engineering and Computer Science \\ ${ }^{2}$ School of Physics and Astronomy \\ Queen Mary University of London \\ London E1 4NS, UK \\ *a.andy@qmul.ac.uk
}

\begin{abstract}
A novel optically-activated antenna array has been proposed, designed, and analysed using photosensitive organic semiconductor poly (3-hexylthiophene) (P3HT, 95\%) and [6,6]Phenyl C61 butyric acid methyl ester (PCBM, 5\%) for S-band applications. A two-patch antenna array, using soda-lime glass as the primary substrate and indium-tin-oxide (ITO) as the ground plane, was designed using the CST Studio Suite. The organic semiconductor heterojunction P3HT:PCBM was employed in the design as a secondary substrate atop the glass. Characterisation of the organic polymer was performed using quasi-optical measurement bench over the WR3 waveguide band to estimate the change in dielectric between dark and active (i.e illuminated), states. This change is taken into account while modelling the antenna array, and the potential beam-steering applications are examined.
\end{abstract}

Keywords—antenna array; beam-steering; optically-activated; organic semiconductor.

\section{INTRODUCTION}

Antenna arrays play a significant role in both radar and satellite communication systems [1]. Phase-shifters have been readily employed in such arrays to implement beam-steering. Conventionally, phase-shifting in antenna arrays is achieved using electronic or mechanical action. However, opticallycontrolled phase-shifters have several advantages over these. Several optically-activated cases of antenna array beam steering have been reported in literature [1-2].

Conventional antenna arrays require separate phase-shifters for each radiating element to off-set the initiation of radiation among neighbouring radiating elements. Nevertheless, these phase-shifting electronic units introduce system-interference, whereas optically-controlled phase-shifters are inherently isolated from the antenna's radiation field. Optically-based phase-control additionally offers a reduced system power budget.

Most optically-controlled phase-shifters are based on the photoconductive effect of inorganic-semiconductor silicon ( $\mathrm{Si}$ ). Light (of 880nm wavelength) is used to tune the conductivity of Si by generating electron-hole pairs which in turn alter the propagation characteristics of the wave propagating in the medium [3-4]. However, Tang et al designed an optically controlled phase shifter employing the organic semiconductor

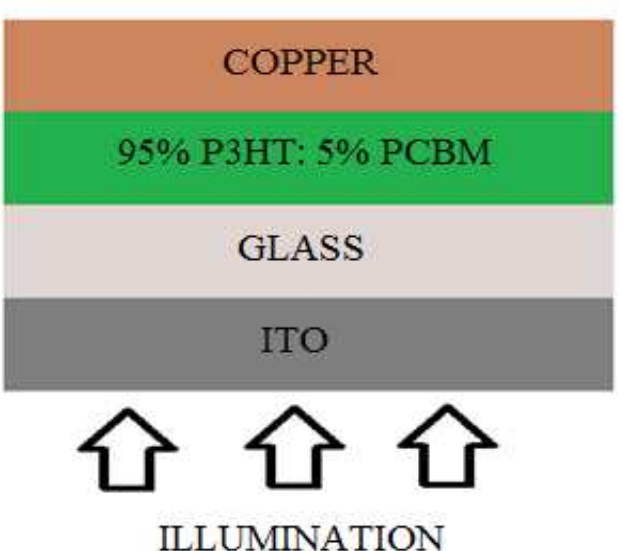

Fig. 1. Schematic diagram of the optically-activated antenna using a glass substrate and indium-tin-oxide (ITO) as the ground plate.

poly(3-hexylthiophene) [5]. This p-type organic semiconductor was demonstrated as a tuneable dielectric. The low mobility of the charge-carriers in organic semiconductors makes them a poor photo-conductive material. Nevertheless, it was verified that these organic polymers measurably change the real part of the permittivity, under optical illumination $(532 \mathrm{~nm})$. The change in permittivity between dark and illuminated (i.e. 'active') states, was estimated at 0.1 .

Photosensitive organic semiconductors such as P3HT are typically used in OLEDs and OPVs. These are low dielectric materials and have a band-gap of $\sim 1.9 \mathrm{eV}$, which is sufficient for the photon energy of white-light to excite the material and generate electron-hole pairs. Additionally, P3HT has high mobility when compared to other photosensitive organic semiconductors [6]. As a result of this high drift mobility of the induced charge carriers of $\mathrm{P} 3 \mathrm{HT}$, it makes this organic semiconductor an appropriate choice for use in photocontrolled phase-shifters.

P3HT is amorphous in nature, for which it has to be dissolved using an organic solvent (e.g. dichlorobenzene or chloroform), heated, and then casted onto a substrate. Methods of deposition onto substrates are either by drop casting or spray coating, depending on the application of the device or the 


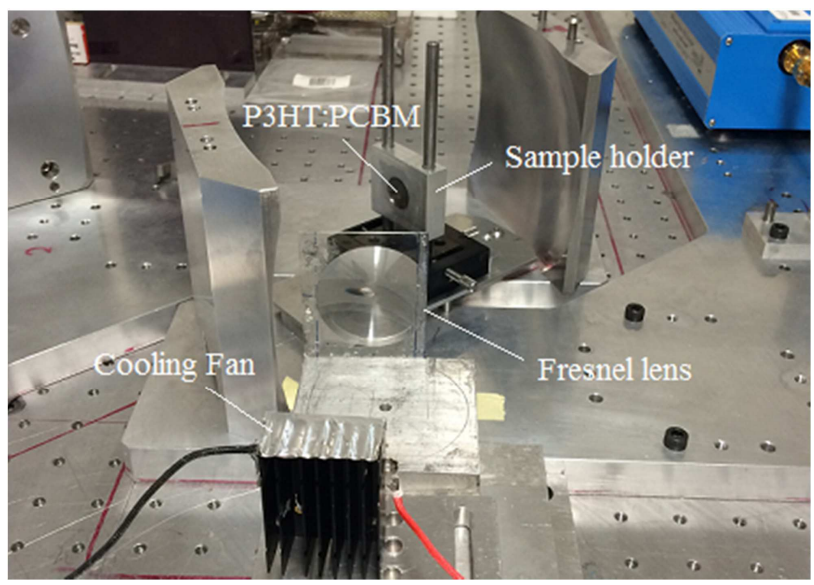

Fig. 2. Measurement setup on the quasi-optical measurement-bench to estimate the change in dilectric constant of the organic polymer blend between dark and lit (i.e. active), states.

sample-holder dropped on. Here we use drop-casting to measure the dielectric constant of the organic polymer and then use that value in the antenna design.

In order to optimally illuminate the organic polymer layer on a substrate, a substrate transparent to white light, sodalime glass, was chosen. The schematic of the layers of the modelled antenna array is shown in Fig.1. The substrate of the antenna is composed of two layers, the first being the optically transparent glass substrate and the second the organic polymer. The ground of the antenna is modelled using indium tin oxide (ITO). This is because ITO is a highly regenerate n-type semiconductor, which is transparent to visible light and has a very low resistivity, therefore acting as a reflector at microwave frequencies.

\section{CHARACTERISATION OF P3HT:PCBM}

A heterojunction using two types of organic semiconductors, 95\% P3HT: 5\% PCBM is used as a tuneablesubstrate top layer in the modelled antenna. This blend has proven to provide the best tuneable range, in terms of change in dielectric between dark and active states of the polymer [7]. When 5\% n-type PCBM is blended with $95 \%$ base polymer $\mathrm{P} 3 \mathrm{HT}$, dissociation of free electrons is promoted, increasing the photo-generated carrier density in the active region of the polymer blend.

\section{A. Sample Preparation}

In order to characterise the polymer blend (95\% P3HT: $5 \%$ PCBM) $50 \mathrm{mg}$ of the concentration was measured using a sensitive beam balance and dissolved in $1.5 \mathrm{ml}$ of organic solvent chloroform. The solution is then allowed to heat on a hot-plate at $60^{\circ} \mathrm{C}$ for two hours for all the P3HT:PCBM granules to dissolve. Once the solution is ready, it is drop-cast onto a suitable sample-holder inside a glove-box.

The sample holder used to mount the organic polymer on the quasi-optic bench is a rectangular aluminium block, with a central circular cut-out of $1.7 \mathrm{~cm}$ diameter. A thin mylar film, permittivity very close to air, is chosen to support the organic film. This $10 \mu \mathrm{m}$ thick film is pasted onto one side of the holder to support the organic polymer solution during drop-



Fig. 3. Dispersive dielectric response of polymer blend over the WR-3 waveguide band estimating the real and imaginary values of the complex permittivity of the organic polymer.

casting. The sample, once drop-cast, needs to stay on the hotplate for 24 hours to dry and stick to the mylar film.

\section{B. Measurements using Quasi-optic bench}

A millimetre-wave measurement bench driven by a VNA [8] is used to characterise the organic polymer heterojunction in its dark and active states. The calibrated complex transmission across the sample is measured, which, together with the thickness of the sample and ABCD matrix theory, is used to extract the real and imaginary part of the complex permittivity of the material under test. Fig. 2 shows the VNAdriven quasi-optic measurement bench setup. The sample holder is mounted onto two pillars on the bench at a point where the beamwidth of the ultra-Gaussian beam field from the transmitting horn antenna is approximately $2 \mathrm{~mm}$. As a result, the central cut-out of $1.7 \mathrm{~cm}$ in the holder allows the incident beam to pass through the sample, with negligible diffraction. Measurements are normalised with respect to transmission through mylar.

The thickness of the sample is measured to be $230 \mu \mathrm{m}$ using profilometry [9]. The mylar film as $10 \mu \mathrm{m}$ as determined by a micrometre screw gauge. In order to excite the photosensitive organic polymer, white light $(532 \mathrm{~nm})$ is used with an irradiance of $65 \mathrm{~mW} / \mathrm{cm}^{2}$ in the sample plane. Estimates of the real and imaginary parts of the complex permittivity of the P3HT-PCBM heterojunction in dark and active states is evaluated (see Fig 3). Although the real and imaginary part of the complex permittivity of the organic blend are measured at a frequency much higher than the operating frequency (S-Band) of the modelled antenna, the change, $\Delta \varepsilon$, between dark and active states in the real part of permittivity, is approximately 0.1. This change in dielectric is used in the CST software [10], while modelling the organic-semiconductor layer, to evaluate the effect $\Delta \varepsilon$ has on the beam pattern of the modelled antenna at S-band.

\section{PHYSICAL PROPERTIES OF GLASS AND ITO}

\section{A. Complex Permittivity of Sodalime Glass}

The complex permittivity of sodalime glass is measured using a split-cylinder resonator [11] driven by a VNA. The 


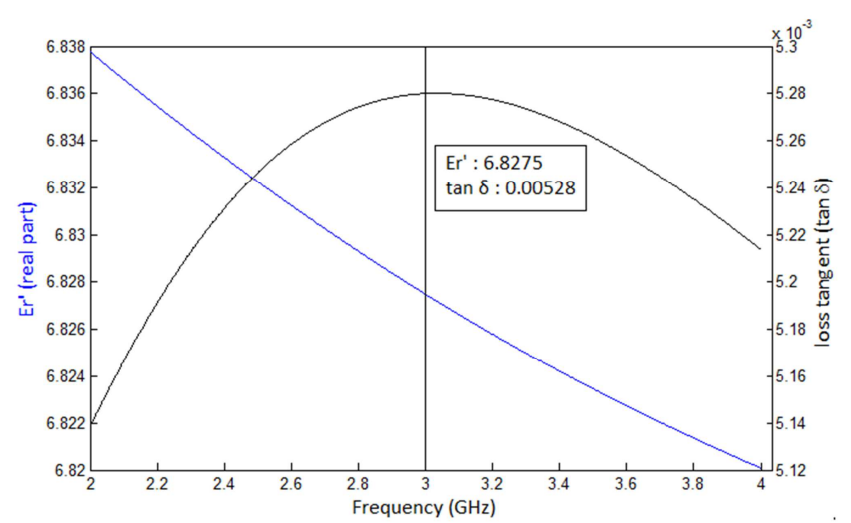

Fig. 4. Measured dielectric response of sodalime-glass over S-band using a Split-Cylinder Resonator and extrapolated using CST.

resonator is designed to resonate at $10 \mathrm{GHz}$ in free-space. The thickness of the glass is measured using a micrometre screw gauge as is $1.11 \mathrm{~mm}$. Depending on the thickness and the dielectric of sample under test, the resonator shifts to a single resonating frequency at the fundamental mode. At the resonant frequency of $8.4 \mathrm{GHz}$ real part of sodalime permittivity is estimated to be 6.82 and loss tangent to be 0.0053. These values are used to model the substrate in CST. The dielectric response is extrapolated to S-Band (see Fig. 4).

\section{B. Conductivity of Indium Tin Oxide (ITO)}

In order to promote accurate modelling of the ITO ground layer in CST, the conductivity of ITO is measured. Measurement of ITO conductivity involved measuring resistivity of ITO using the well-known four-point probe method. This method works with four equi-distant, linear probe points. The two outer points represent the dipole, and the two inner, the current, I. In the calculation of the resistivity of the material under test, the voltage, $\mathrm{V}$, between the two inner probes is measured.

ITO-coated sodalime glass is used in the antenna design as a substitute to the conventional dielectric-copper model. The thickness of the ITO coating on the glass-substrate is measured by etching and forming a pattern of ITO on the glass substrate to measure the difference between the two layers. For a sample thickness $\mathrm{w}$ much smaller than the probe separation $\mathrm{s}$, resistivity, $\rho$, of the sample can be directly calculated by $\rho=$ $(\pi / \ln 2) \mathrm{w}(\mathrm{V} / \mathrm{I})$. The measured thickness was $110 \mathrm{~nm}$ and the corresponding resistivity $1.5 \times 10^{-6} \Omega \mathrm{m}$.

\section{ANTENNA DESIGN}

A two-patch antenna for S-band application was designed using CST. The substrate of the antenna array is modelled as a multi-layered structure with the organic polymer layer stacked on top of the soda-lime glass sample. The antenna design was selected from previous work, and modified according to the new substrate definition [12]. The modelled structure consists of four layers; copper, organic polymer, sodalime-glass and ITO, respectively. The substrates and ground plane are modelled using the measured parameters estimated in Sections II and III. Measured values of conductivity and thickness of ITO are used to model the ground plane.

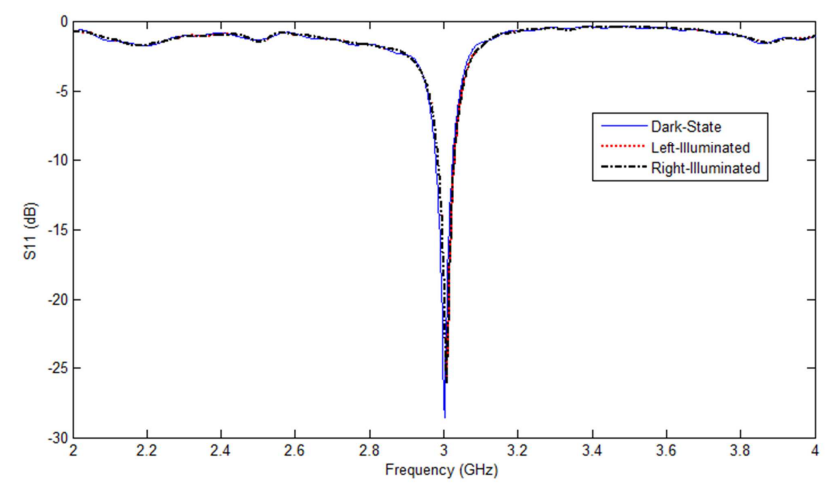

Fig. 5. Simulated return loss of the two-patch antenna array showing the dark and active state resonant frequencies.

The organic layer on the glass-substrate was modelled using a thickness of $0.36 \mathrm{~mm}$, chosen according to the practical useful plasma-depth of the layer. This layer was placed on top of the glass, covering the whole area $\left(70 \times 90 \mathrm{~mm}^{2}\right)$. In practice, this design will be fabricated by vacuum depositing aluminum or copper onto the organic layer.

The return loss of the modelled antenna is analysed (see Fig. 5). In the dark-state of the antenna, the impedance is matched at $-30 \mathrm{~dB}$. The left and right side illuminating conditions are modelled by changing the dielectric properties of the respective side. Measured values of $\varepsilon_{\mathrm{r}}=3.0, \tan \delta=$ 0.06 for dark conditions and $\varepsilon_{\mathrm{r}}=2.9, \tan \delta=0.089$ for illumination are considered as inputs. Both the rightilluminated and left-illuminated conditions show impedance matching.

Figs. 6 (a) and (b) shows the surface current distribution of the two-patch antenna array for left-patch illuminated and right-patch illuminated conditions at $3 \mathrm{GHz}$, respectively. Under left-illuminated condition, as the phase of excitation advances, the surface current distribution at the left patch is different from the right, and vice versa for the rightilluminated condition. This demonstrates the fact that there is an uneven distribution in the magnitude of the surface current vector among the patches during illumination due to the phase lag. A symmetrical beam steer of $5^{\circ}$ off boresight was observed for both illuminated conditions from the simulated farfield radiation pattern (see Fig. 7).

\section{DISCUSSION OF RESULTS}

Although the complex permittivity measurements for the organic polymer blend were taken at a much higher frequency compared to the operating frequency of the modelled device, it is estimated that the change is permittivity between dark and active states remains approximately constant in frequency between $\mathrm{S}$ and WR-3 bands. This provided the data input required to show the proof-of-concept for beam steering with such a phase-shifting device. In addition, the antenna design also demonstrated that with a small change in the real part of the permittivity of the substrate it is possible to generate a significant beam steer $\left(5^{\circ}\right.$ in this case), using an effective phase-shifting design. 


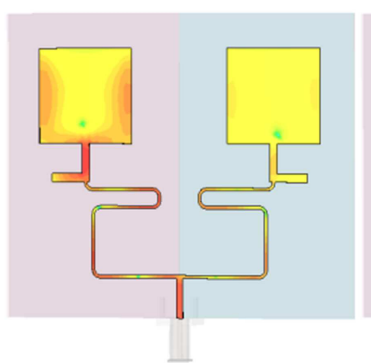

(a)



(b)

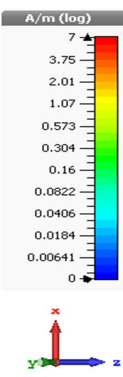

Fig. 6. Simulated average surface current distributions: (a) left-side illuminated condition; (b) right-side illuminated condition.

Farfield Realized Gain Abs (Phi=90)

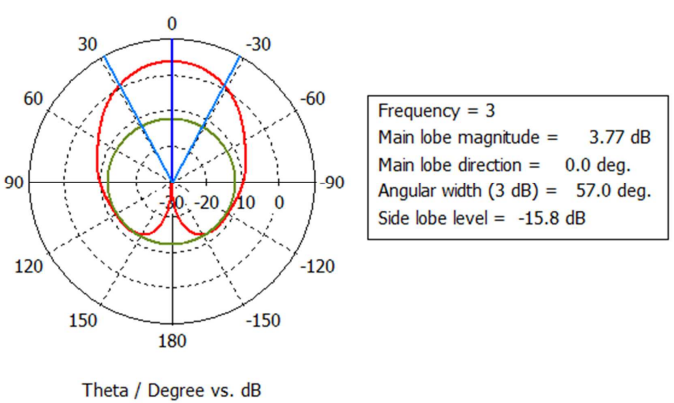

(a)

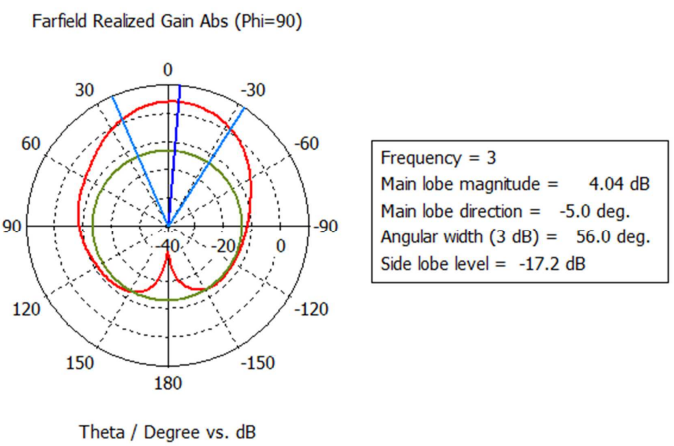

(b)

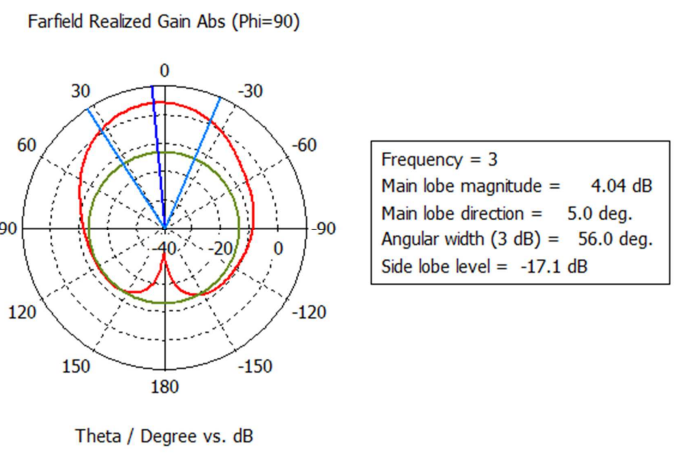

(c)

\section{CONCLUSION}

A novel optically-activated antenna array using photo-activated organic semiconductor (P3HT: PCBM) has been presented. The primary objective of the proposed design was to justify the concept of beam-steering using a photo-sensitive tuneable dielectric material. The optically-tuneable organic polymer was characterised using a mm-wave measurement bench. The proposed design generated a $5^{\circ}$ beam steer off boresight at $\mathrm{S}$ band. A potential future application for the proposed system is the employment of the device for point-to-point beam alignment for detection and steering in data-centre highcapacity communication links.

\section{ACKNOWLEDGMENT}

The authors would like to thank the Antenna Laboratory Manager Dr. Massimo Candotti, and also the Centre for Condensed Matter and Materials Physics Technicians, Mr. George Nevill and Mr. Geoffrey Simpson, for providing equipment and assistance during measurements.

\section{REFERENCES}

[1] M. E. Khaldi, F. Podevin, and A. Vilcot, "Optically controlled microstrip phased arrays," in Microwave Photonics, International Topical Meeting on, Oct. 2005, pp. 193-196.

[2] C. Panagamuwa, A. Chauraya, and J. Vardaxoglou, "Frequency and beam reconfigurable antenna using photoconducting switches," IEEE Trans. Antennas Propag., vol. 54, no. 2, pp. 449-454, 2006.

[3] M. E. Khaldi et al., "Improvement of performance of optically controlled microstrip phase shifters," IET Microw. Antennas Propag., vol. 1, pp. 427-432, Apr. 2007.

[4] H. P. Li and K. T. Chan "An improved optically controlled phase shifter on si wafer" Microwave Opt. Technol. Lett., vol. 44, pp. 540-541, Mar. 2005.

[5] H. Y. Tang, R. S. Donnan, and T. Kreouzis, "An optically controlled phase shifter employing the organic semiconductor poly (3hexylthiophene)," Applied Physics Letters, vol. 91, 202101-3, 2007.

[6] K. M. Coakley et al., "Enhanced hole mobility in regioregular Polythiophene infiltrated in straight nanopores," Advanced Functional Materials, vol. 15, pp. 1927-1932, 2005.

[7] A. S. Andy et al., "Optically-tunable organic semiconductor heterojunction P3HT-PCBM for millimeter-wave applications," in Infrared, Millimeter, and Terahertz waves (IRMMW-THz), 40th International Conference on, Aug. 2015, pp.1-2.

[8] Keysight Technologies N5244A PNA-X Network Analyzer.

[9] Bruker DektakXT Stylus Profiler.

[10] CST Studio Suite: https://www.cst.com/

[11] Keysight Technologies 85072A 10-GHz Split Cylinder Resonator.

[12] A. Andy et al., "An optically controlled phase shifter for antenna array beam steering," in Antennas and Propagation (EuCAP), 9th European Conference on, Apr. 2015, pp.1-4.

Fig. 7. Simulated farfield radiation pattern of the modelled antenna: (a) darkstate; (b) left-illuminated condition; (b) right-illuminated condition. 